\title{
XML for Detector Description at GLAST
}

\author{
J. Bogart (SLAC, Menlo Park) \\ D. Favretto, R. Giannitrapani (Università di Udine, Udine) \\ Abstract \\ The problem of representing a detector in a form which is accessible to a variety of \\ applications, allows retrieval of information in ways which are natural to those applications, \\ and is maintainable has been vexing physicists for some time. Although invented to address \\ an entirely different problem domain, the document markup meta-language XML is well- \\ suited to detector description. This paper describes its use for a GLAST detector. [f]
}

Keywords: detector, GLAST, XML

\section{Introduction}

For any experiment an accurate description of its apparatus is essential. Some features of HEP experiments make this goal especially difficult to achieve:

- Detectors are large and complex.

- Experiments tend to be long-lived. Meanwhile prototypes, actual and simulated, must be supported; production detectors undergo upgrades; software technology evolves.

- Several applications need access to the detector description.

Hence the scheme used for detector description must be flexible and versatile. Any information potentially of interest to more than one application, in particular all geometric parameters and relationships, must exist as data, not code.

\section{$1.1 \mathrm{XML}$}

XML[5] was originally intended as a means of designing web document types so that any part of the content of the document, not just formatting information, could be tagged and thus made readily available to programs. However, it quickly became clear that such "documents" could include databases or structured messages as well as more typical document content. As a result of its wide appeal, including many commercial applications, high-quality tools for XML soon became available and continue to evolve rapidly. XML is not only a suitable vehicle for detector description now, but is well-supported and likely to remain so for years to come. It is therefore not surprising that XML has gained broad popularity in the HEP community; many experiments have adopted it as their geometry persistency mechanism ${ }^{2}$

\section{$1.2 \quad$ GLAST LAT Specifics}

Our task is to provide a description for the Large Area Telescope (LAT), the primary instrument of the two composing the GLAST observatory scheduled for launch in 2006. It is a small, relatively simple HEP detector designed to detect gamma rays over a large energy range. Active volumes are practically all boxes, channel counts in two of three subdetectors are modest, and tolerances are generally less stringent than for large HEP experiments.

In other respects the LAT detector and the environment in which its software is being developed are typical of HEP. The usual collection of programs - simulation, reconstruction, calibration procedures, etc. - are all necessary. Most are $\mathrm{C}++$ applications being structured using the Gaudi framework which must run on multiple platforms (Windows, Linux, Solaris). The architecture must support laptops disconnected from the net as well as less constrained computing environments. Tools have to survive for the lifetime of the project, at least a decade.

\footnotetext{
${ }^{1}$ Work supported by the Department of Energy, contract DE-AC03-76SF00515

${ }^{2}$ In the last two years three specialized workshops have been dedicated to discuss this topic among different experiments; see, for example [3].
} 


\section{Implementation}

There are three major pieces to our XML description schema, currently implemented as a DTD known as GDD, and to the software interpreting it. At its heart is a method for describing geometric solids and their relative positions. The second piece, a mechanism for definition, manipulation and substitution of symbolic constants, was designed to aid in maintenance and comprehensibility of the geometry description. Finally, to divorce all potentially shared parts of detector description from any single application, GDD includes facilities to define and manipulate identifiers associated with parts of the detector.

\subsection{XML Parser}

We use facilities of XML to enforce constraints on the detector description whenever possible, hence we need a validating parser. It must be compatible with $\mathrm{C}++$ since vital applications such as simulation are written in $\mathrm{C}++$. Furthermore, parser support for the Document Object Model (DOM) 2] interface saves us from having to design our own generic XML in-memory representation [3. We have chosen the open-source Xerces-c parser 清. It has all required features, and additionally has a large, supportive community of users and developers.

\subsection{Geometry}

\subsubsection{XML description}

The plethora of possible clients of the GLAST geometry has lead to a quite general design for those XML elements in GDD dedicated to geometry, mainly inspired by AGDD[6], the ATLAS DTD, which in turn owes much to simulators such as GEANT.

The DTD defines volumes and positioning elements. Volumes may be geometric primitives, like boxes or tubes, or composites constructed of several other (primitive or composite) volumes, nested to arbitrary depth. Positioning elements describe the relative positions of child volumes within their parent.

\subsection{2 detModel and clients}

In order to isolate client applications from XML itself, we have developed a $\mathrm{C}++$ layer called detModel, a class hierarchy used by GLAST applications to access the geometry description of the detector stored in an XML file. Any software component needing geometrical information accesses it via detModel; the details of the XML description and the parser interface are hidden.

To develop this package, our analysis started from GDD and its hierarchical structure. $\mathrm{XML}$ is not object oriented, but there is a natural mapping from GDD elements to classes connected by "has-a", "is-a" and "use-a" relations, suggesting a possible hierarchy for these classes. This internal structure is quite independent of client nature and access, for which we have added a series of management classes and query functionalities on the geometric hierarchy. From the start we have tried to implement a certain degree of modularity; in this way every client can instantiate and access only the part of GDD that it really needs.

Throughout the design and implementation of detModel we used design patterns [1] extensively. In particular the manager class GDDmanager, the gateway to the full hierarchy, is a singleton; moreover we have separated the construction of the geometry representation from the representation itself by adopting a builder pattern. In this way, for example, we have decoupled detModel (and all its clients) from the specific XML parser used, leaving open the option to change it in future. We also adopted the visitor pattern as the basic mechanism for accessing the full hierarchy; every client needs only to implement a concrete visitor, implementing whatever

\footnotetext{
${ }^{3} \mathrm{~A}$ larger experiment might balk at the memory usage of the DOM, but it is unlikely to be an issue for us.
} 
functionalities it requires. For clients that do not need to access the full hierarchy, but only a part of it we have also implemented some basic direct query mechanisms.

With both the visitors mechanisms and the direct query methods of the GDDmanager public interface it is quite easy to quickly implement specific requirements for a broad collection of clients. We have already developed a VRML client for graphical representation of the geometry, a GEANT4 client that can use detModel to instantiate the geometry in the Monte Carlo simulation (including sensitive detector information contained in GDD) and a prototype ROOT visitor to access the geometric information of GLAST inside analysis and event display programs. See [8] for sample images produced by these clients. For the future we are working on a HepRep client of detModel for some possible links with WIRED and JAS clients and a GISMO client (the Monte Carlo toolkit currently used by the GLAST collaboration).

\subsection{Constants}

In describing even a relatively small detector like the LAT naively with XML, one soon mourns the absence of standard conveniences, such as support for constant names and arithmetic. Dimensions are often repeated; offsets are commonly calculated from other values. If all such values are entered as literal constants the description quickly becomes unmaintainable.

\subsubsection{XML definitions}

Although XML has no built-in arithmetic one can define elements to specify the arithemetic to be done by co-operating applications. We define XML elements for "primary" and "derived" constants, for references to constants, and for standard arithmetic operations. Primary constants have only a name, a value, type information, and a descriptive comment. Derived constants may have element content consisting of arithmetic elements and references to other constants. Other XML elements, such as those describing volumes of a particular shape, have been modified to accept either a literal value for, e.g., a radius, or a reference to a constant. Constant names are IDs in the XML sense and references to them are IDREFs, so validating parsers enforce uniqueness of such names and verify that all references resolve properly.

\subsubsection{Supporting software}

Utility $\mathrm{C}++$ classes have been written to evalute derived constants, optionally replacing their element content with a value attribute, and to substitute a value for a reference to a constant, doing the evaluation first if necessary. These utilities may be invoked directly by programs such as Simulation. A stand-alone program using these utilities takes an arbitrary XML document as input and outputs an equivalent document with all expressions evaluated and all references replaced with values. For production running this preprocessing saves resources, most notably the memory required for the DOM representation, which is considerably smaller and less nested for the preprocessed document.

The constants have interest in their own right, both to programs which need access to a particular parameter apart from the complete geometry description, and to humans in search of a reliable source of documentation. $\mathrm{C}++$ classes have been written to "serve" the constants via both visitor and query interfaces, as has a stand-alone visitor client which produces html tables of constants, readily accessible to the full collaboration for review.

\subsection{Identifiers}

Several clients of detector description need a way to identify individual volumes, both for internal use and to label information, such as hits, which may be generated by one application and read by another. No single labeling scheme can gracefully accomodate all clients. An 
analysis client may only be concerned with read-out volumes whereas Simulation must be aware of all volumes. GDD supports multiple labelings and, where feasible, transforms between them.

\subsubsection{XML definitions}

Positioning elements may include an idField, which is composed of a fieldname and value. An identifier for a volume is the concatenation of the values of its own and ancestor idFields.

In order to validate an identifier or manipulate identifiers as a group, we use an identifier dictionary. A dictionary specifies legal sequences of fieldnames and constraints on values in a particular context. Dictionaries may be used to constrain sets of identifiers (such as readout identifiers) other than those coming from positioning elements. Conversions taking identifiers from one dictionary to another are specified using an id converter. Values occurring in dictionaries or converters can be literals or references to be resolved by substitution.

\subsubsection{Supporting software}

GDD imposes a hierarchical structure on the set of identifiers belonging to a given dictionary, but does not enforce certain uniqueness properties. Id dictionary classes have a validation function to ensure, e.g., that any allowed sequence of field values has a uniquely-associated sequence of fieldnames. Individual identifiers may be checked for consistency with a dictionary.

\section{Status and Conclusions}

The current description of the LAT using GDD, including a compatible id dictionary, describes all active and some structural components. Remaining components will be added as needed. Except for id conversion, all fundamental services needed to interpret a GDD document have been implemented. Several key clients are up and running; others are in the works.

As the LAT description grows in size and complexity, we need to monitor potential resource bottlenecks, particularly memory. Maintenance of the description files is more immediately troublesome. The ID/IDREF mechanism is heavily used in GDD, which leads to problems in name selection and management: ID values must be a unique within the document. A move to XML Schema, a newer alternative to DTDs, would help.5 GDD documents are fragile in the sense that small change in the design of the detector can entail large changes in the document. However, if the information in the document were kept in code instead such changes would be significantly more disruptive. In summary, using XML to describe detectors, while not ideal, appears to be workable, and probably significantly superior to older technologies.

\section{References}

[1] E.Gamma et.al, Design Patterns: Elements of Reusable Object-Oriented Software, Addison-Wesley 1994

[2] DOM Level 2 Core Specification, http://www.w3.org/TR/DOM-Level-2-Core/

[3] HEP Detector Description Workshop (CERN), http://doc.cern.ch/age?a01380

[4] Xerces C++ Parser http://xml.apache.org/xerces-c/index.html

[5] XML 1.0 Specification, http://www.w3.org/TR/REC-xml

6) http://atlas.web.cern.ch/AtIas/GROUPS/DATABASE/detector_description/

7 http://www-glast.stanford.edu/mission.htmI

[8] http://wWW.fisica.uniud.it/ riccardo/research/glast/gallery

\footnotetext{
${ }^{4}$ The concept and name are due to members of the ATLAS AGDD Working Group.

${ }^{5}$ XML Schema has other advantages which space does not permit us to describe.
} 